\title{
Agôn
}

Revue des arts de la scène

7| 2015

La Distribution

\section{La distribution des rôles dans la tragédie athénienne}

\section{Diomedes Gariazzo Lechini}

\section{(2) OpenEdition}

Journals

Édition électronique

URL : http://journals.openedition.org/agon/3218

DOI : 10.4000/agon.3218

ISSN : 1961-8581

\section{Éditeur}

Association Agôn

\section{Référence électronique}

Diomedes Gariazzo Lechini, « La distribution des rôles dans la tragédie athénienne », Agôn [En ligne], 7 | 2015, mis en ligne le 17 juillet 2015, consulté le 19 avril 2019. URL : http://journals.openedition.org/ agon/3218; DOI : 10.4000/agon.3218

Ce document a été généré automatiquement le 19 avril 2019.

Association Agôn et les auteurs des articles 


\title{
La distribution des rôles dans la tragédie athénienne
}

\author{
Diomedes Gariazzo Lechini
}

\section{Trois acteurs}

1 Des sources anciennes (plus tardives que les tragédies que nous conservons) témoignent du nombre d'acteurs qui jouaient dans une tragédie et de l'évolution qui porta à ce nombre. En nous basant sur ces sources, nous proposerons une reconstruction de l'évolution de la tragédie attique quant à ses acteurs.

2 À l'origine, les tragédies n'étaient interprétées que par un seul acteur, le poète, qui était aussi compositeur musical, chorégraphe et metteur en scène de la pièce, c'est-à-dire son créateur unique. Nous n'avons aucune tragédie appartenant à cette première étape. Dans cette période, entre les dernières années $d u \mathrm{vI}^{\mathrm{e}}$ et les premières décennies $\mathrm{du} \mathrm{v}^{\mathrm{e}}$ siècle avant J.C., la tragédie était un spectacle où un poète et son chœur parlaient et chantaient en représentant des personnages ${ }^{1}$, et rien d'autre. Dans un deuxième stade d'évolution, on eut l'idée d'ajouter un deuxième interprète soliste (selon les sources, on doit cette innovation à Eschyle); cela permettrait d'ajouter des scènes dialoguées au spectacle. Pour cette période, nous avons des témoignages: Les Perses, Les Sept contre Thèbes et Les Suppliantes d'Eschyle appartiennent toutes à ce stade d'évolution. Cette innovation a pu naître de la pure invention d'un poète (que ce soit effectivement Eschyle ou pas ${ }^{2}$ ) ou d'une évolution générale dans la façon d'envisager le spectacle, mais en tout cas elle n'aurait pu rester dans le domaine de l'innovation personnelle : le contexte des concours dans lequel se déroulaient les festivals dramatiques exigeait qu'elle fût acceptée de façon officielle et qu'elle devînt la nouvelle règle du jeu. Désormais la tragédie était un spectacle où le poète se faisait accompagner par un autre interprète pour alterner avec le chœur dans une suite de discours, de dialogues et de chants ; et rien d'autre. Quelques décennies plus tard, on eut l'idée d'ajouter encore un nouvel interprète soliste (innovation attribuée à Sophocle, ou encore à Eschyle ${ }^{3}$ ), sans doute parce que la possibilité d'écrire des scènes avec trois personnages parlants intéressait, mais aussi parce que trois acteurs 
permettraient la mise en jeu d'un nombre total de personnages plus important, tout en apportant plus de dynamisme aux entrées et aux sorties. Cette innovation fut aussi acceptée et devint la nouvelle règle du jeu. Peu de temps après, l'abandon de la scène par le poète 4 fit que la tragédie fût dès lors un spectacle poétique interprété par trois acteurs et un chœur. Et elle ne fut jamais autre chose. Les Grecs antiques n'ayant jamais connu un théâtre qui reposait sur une correspondance entre le nombre d'acteurs et le nombre de personnages, ni qui mettait en jeu plus de trois acteurs, imaginer une règle qui restreigne à trois un nombre d'acteurs potentiellement supérieur apparaît comme un anachronisme. Plus qu'une règle, c'était une caractéristique essentielle du genre. Une lecture des sources devrait pourtant suffire à certifier ce point: elles expliquent comment le nombre d'acteurs est passé d'un à trois, mais ne nous disent pas qu'il y avait une règle qui empêchait l'emploi de davantage d'acteurs.

Pourquoi le nombre d'acteurs s'est-il arrêté là ? Dans la forte incompréhension dont la «règle des trois acteurs » a été l'objet, différentes hypothèses ont été avancées pour justifier cette convention incommode. Parmi les plus improbables, mentionnons celle qui consiste à en attribuer la responsabilité aux prêtres de Dionysos (dieu patron des festivals), en raison d'un soi-disant désir de "conservatisme» du genre tragique. Une autre justification, souvent convoquée et jouissant de davantage de crédit, me paraît en revanche intéressante à réfuter ici. On a avancé que des raisons économiques auraient favorisé cette restriction. Les acteurs, à la différence des choreutes, étaient en effet payés par la cité, et cela a semblé une raison suffisante pour restreindre leur nombre. Or, il se trouve que les choreutes, payés par les chorèges (qui, nommés par les autorités, finançaient les spectacles) avaient eux aussi un nombre limités. De surcroît, la cité aurait très bien pu payer les trois acteurs principaux tout en laissant les chorèges libres de payer autant d'acteurs mineurs que les poètes souhaitaient. En effet, les chorèges faisaient des frais beaucoup plus considérables, y compris en engageant un nombre illimité de figurants, ces derniers pouvant d'ailleurs être utilisés pour la représentation de personnages qui, dans une scène donnée, font une apparition muette (quand ils parlent, les personnages sont pris en charge par des acteurs) ${ }^{6}$.

4 La réponse à la question initialement posée - pourquoi le nombre d'acteurs s'est-il arrêté à trois? - ne peut être que très simple: parce que cela semblait assez, et parce que davantage aurait semblé trop. Si l'on sépare le plan strictement scénique - qui n'affecte que le nombre des personnages parlant dans chaque scène - du plan de la composition dramaturgique - qui est conditionnée par le nombre total des acteurs -, nous pouvons faire deux constats. Le premier, sur le plan scénique, est que les poètes n'ont apparemment pas eu d'intérêt pour des scènes avec plus de trois personnages parlants : trois, c'était déjà beaucoup, comme tend à le démontrer l'exploitation très mesurée que les poètes font de ce type de scène. Le second, sur le plan de la composition dramaturgique et du jeu des acteurs, est que plus d'acteurs auraient rendu les choses «trop faciles » et donc moins intéressantes. La tragédie avait depuis très tôt (peut-être depuis toujours) impliqué l'interprétation de plusieurs personnages de la part de l'acteur ou des acteurs ${ }^{7}$. Un des intérêts du spectacle tragique résidait sans doute dans cette différence entre le nombre des personnages et celui des acteurs, différence qui demandait de la souplesse de la part des interprètes et de l'habileté dramaturgique de la part du poète. L'ajout d'acteurs supplémentaires aurait rendu ces arrangements plus faciles. Mais voulait-on qu'ils deviennent plus faciles? La limitation du nombre d'acteurs a son origine dans les racines mêmes du genre et elle est devenue une convention que jamais personne 
ne songea à transgresser. Face à une question du genre « Pourquoi dans le football il n'y a-t-il que onze joueurs par équipe ? ", une réponse très naturelle serait «Parce que c'est comme ça, le football est comme ça ». Eh bien, la tragédie aussi « c'était comme ça ».

5 En effet, la tragédie est dans un certain sens plus proche du sport que du théâtre moderne : que ce soit aux Dionysies ou dans d'autres festivals, la tragédie était associée à des concours où la victoire était essentielle. Et là où il $\mathrm{y}$ a des concours, il $\mathrm{y}$ a des critères fixes, l'intérêt des confrontations résidant dans la façon qu'on a de faire ce que l'on fait à l'intérieur d'un cadre donné. Les concurrents doivent montrer leur habileté dans un cadre assez strict de conventions, définies par un règlement ou par la tradition et la coutume. Sous de telles conditions, la simple volonté d'un poète n'aurait pas suffi pour ajouter un quatrième acteur : il fallait changer les conventions du genre et que tous les concurrents s'y adaptent. Sans un tel changement dans les conventions, l'innovation serait impensable, de la même façon qu'il serait impensable de mettre en scène une pièce en prose plutôt qu'en vers, ou de faire varier le nombre - qui lui aussi était fixe - des choreutes. Ces bases devaient être les mêmes pour tous les concurrents. On a souvent suggéré que l'ajout d'un quatrième acteur était nécessaire pour des pièces où les acteurs auraient dû trop se dépêcher pour changer de costume, comme si le poète avait pu ajouter un acteur de façon sporadique à chaque fois qu'il le voulait ${ }^{8}$. Ce serait comme imaginer un entraîneur de football qui aurait la possibilité d'ajouter un douzième joueur dans des « situations difficiles».

6 Quelles sont les implications de cette convention, de ce trait caractéristique de la tragédie antique? Tout d'abord, que le poète ne peut pas composer de scènes à plus de trois personnages parlants. Plus encore, il doit prendre en considération la distribution des rôles à tout moment, pour agencer les entrées et les sorties de façon à rendre possible la présence de tel ou tel acteur dans une scène donnée. Il ne faut surtout pas penser qu'il adapte une pièce abstraite pour se conformer à une règle pratique imposée ${ }^{9}$. Mais ce qui a du mal à être pris en considération dans la façon la plus répandue d'apprécier la tragédie, c'est surtout le fait que l'agencement des trois acteurs pouvait constituer, en tant que tel, un intérêt pour le public. C'est-à-dire que le poète pouvait composer des scènes de façon à impressionner le public quant à son habileté pour exploiter au mieux ses trois acteurs.

7 Concernant les agencements requis et justifiés par le nombre limité d'acteurs, nous avons vu que le nombre de personnages était plus élevé que celui des acteurs, souvent beaucoup plus : il est donc évident que l'un, les deux voire les trois devaient jouer plus d'un rôle. Attribuer deux personnages au même acteur pouvait avoir un effet spécial exploité par les poètes; cela semble être le cas des Trachiniennes de Sophocle, où le protagoniste jouait sans doute Héraclès dans l'exode et sa femme Déjanire pendant le reste de la pièce ${ }^{10}$. Par ailleurs, pour pouvoir réutiliser un acteur, le poète devait faire sortir des personnages pour des raisons qui, du point de vue de la vraisemblance dramatique, nous semblent parfois recherchées ou forcées ; or nous pouvons aussi y voir une occasion pour le poète de montrer son habileté pour concilier conventions et dramaturgie. Une autre stratégie scénique consiste à attribuer un même personnage à plusieurs acteurs tout au long de la pièce. Bien que cette hypothèse soit celle qui a le plus suscité l'opposition des philologues, nous défendons l'idée que cela donnait une souplesse majeure dans l'agencement des scènes, permettant notamment que l'acteur ayant le plus de talent et d'importance reprenne un rôle qu'il n'avait pas joué jusque-là au moment de la pièce où ce rôle comprend une partie d'importance majeure. 


\section{Acteur principal} travail. Comme nous l'avons vu, à l'origine, les tragédies étaient jouées uniquement par le poète. Cela ne laisse que deux possibilités : soit les tragédies ne comptaient qu'un seul personnage parlant, soit elles en comptaient plusieurs, mais qui se succédaient. Dans le deuxième cas, le poète-acteur devait quitter la scène pour changer de masque et de costume pendant les chants du chœur. Dans un deuxième temps, les rôles se partageaient entre le poète et un second acteur que nous pouvons considérer comme un " assistant ». Plus tard, Sophocle aurait ajouté le troisième acteur, avant de quitter lui-même la scène nous avons donc le poète-acteur avec deux assistants, puis trois acteurs dirigés par le poète. Suivant l'exemple de Sophocle, Euripide n'a apparemment jamais joué dans ses pièces, ce qui semble indiquer que les poètes se sont définitivement éloignés de la scène.

Ce fonctionnement entre l'acteur-poète et les acteurs assistants semble avoir laissé sa trace dans la collaboration entre le poète et les acteurs une fois que celui-là quitta la scène. En effet, dans un premier temps, les acteurs continuèrent à être associés à un poète, d'une façon non officielle. Certaines sources nous font ainsi comprendre que les poètes avaient leurs acteurs de confiance, pour lesquels ils créaient les rôles. Ce principe de collaboration fut cependant remis en question par l'évolution que connut le système des concours. En effet, tant que les poètes jouèrent eux-mêmes leurs pièces, le seul prix qu'on attribuait dans les concours de tragédie était celui de la meilleure tétralogie. Mais quand les poètes abandonnèrent le métier d'acteur, on ne tarda pas à vouloir évaluer le travail des acteurs principaux, séparément du reste du spectacle, en remettant un prix d'acteur (vers l'année 450 av. J.-C.). Quelques années plus tard, on brisa le lien qui existait entre les acteurs et les poètes: au lieu d'être directement choisis par les poètes, les acteurs leur étaient attribués par la voie d'un tirage au sort. Plus tard encore (peut-être quelques décennies, mais la marge de temps est incertaine), on voulut rendre les concours de tétralogies et d'acteurs à la fois plus indépendants entre eux et plus égalitaires; pour cela, le système de tirage au sort fut abandonné : les acteurs jouaient une pièce de chacun des trois poètes concurrents.

Or toutes ces dispositions semblent n'avoir été appliquées qu'à un seul des trois acteurs qui composaient la distribution des pièces: celui que l'on considère comme l'acteur principal. En effet, la trace du poète-acteur n'a peut-être jamais disparu, car il y a toujours eu un acteur principal sur lequel pesait la responsabilité majeure du jeu de la pièce et qui était le seul candidat pour la remise du prix d'acteur ${ }^{11}$. De lui on disait qu'il jouait les "premières parties", qu'il était le "protagoniste" ("le premier dans le concours »), ou simplement qu'il était « l'acteur » de la pièce. Une différence importante devait donc exister entre le poids du jeu de cet acteur principal et celui des autres acteurs. Quelques sources semblent impliquer qu'une hiérarchie existait aussi entre les deux autres acteurs, mais cela n'est pas certain ${ }^{12}$; aucune différenciation formelle ne semble avoir existé entre eux (aucun ne participait au concours d'acteurs).

Ce que nous devons retenir avec le plus d'attention à propos du système de travail des acteurs, c'est le fait qu'il y a toujours eu un interprète plus important que les autres : auteur-poète tout d'abord, puis acteur principal en collaboration avec le poète, puis chef de troupe $^{13}$. Les termes "protagoniste, deutéragoniste, tritagoniste ", " premières, deuxièmes, troisièmes parties ", etc., dont l'emploi est d'ailleurs assez incertain, doivent 
être interprétés en fonction de cette hiérarchie d'acteurs et non pas de l'importance d'un rôle: le "protagoniste» n'est pas un personnage, mais l'acteur qui joue les parties principales de la pièce.

\section{Les rôles}

12 Passons maintenant aux rôles qui doivent être distribués entre ces deux ou trois acteurs. Nous ne ferons pas de référence, cela va de soi, aux personnages qui ne parlent pas dans toute la pièce et qui sont donc incarnés par un figurant ${ }^{14}$. Quant au chœur, il n'est qu'un personnage collectif et aucun de ses composants ne peut être individualisé ${ }^{15}$.

Combien de personnages une tragédie grecque peut-elle comporter? Le nombre est libre et varie beaucoup. Parmi les pièces conservées, le minimum est de deux personnages (une pièce), et le maximum onze (deux pièces) ${ }^{16}$. La seule pièce qui a le même nombre de personnages que d'acteurs est celle qui en a deux, Les Sept contre Thèbes d'Eschyle. Quand le nombre de personnages devient très élevé, il est évident que des arrangements assez sophistiqués devaient être agencés par le poète pour les distribuer entre les trois acteurs. Pour un spécialiste attentif à la dramaturgie des pièces, le fait que les acteurs de tragédie étaient très peu nombreux devrait être une évidence déduite, d'une part, des entrées et des sorties des personnages et, d'autre part, de l'absence de scènes à plus de trois personnages parlants. Or ce n'est pas l'évidence dramaturgique qui a fait connaitre cette convention : ce sont des sources explicites. Quelle position aurait pris la critique si nous n'avions pas eu ces sources, qui sont d'ailleurs très maigres? La question peut sembler étrange, mais elle peut nous aider à comprendre l'attitude des spécialistes par rapport à une autre convention du théâtre grec: le fait qu'un même personnage pouvait être joué, dans des parties différentes d'une tragédie, par des acteurs différents. À partir de l'étude d'Édipe à Colone, ce fait devrait être accepté comme une certitude, mais aucune source explicite ne nous renseigne sur cette particularité. La critique a donc ignoré cette possibilité de façon presque unanime. Cependant, une étude attentive de la dramaturgie des pièces aurait pu la conduire dans ce sens.

EEdipe à Colone n'est pas une pièce avec un nombre particulièrement élevé de personnages: elle en compte huit, comme douze autres tragédies. Mais elle a la particularité d'avoir une suite d'entrées et de sorties qui rend impossible une distribution avec trois acteurs sans le partage d'un personnage entre les acteurs. En effet, les spécialistes ont observé que Thésée devrait être joué par deux, voire par trois acteurs pour qu'on puisse reconstruire une distribution vraisemblable. Cela a révolté plus d'un philologue, et on a trouvé des alternatives fort hardies, parmi lesquelles, bien sûr, on compte l'ajout d'un ou plusieurs acteurs complémentaires. Aujourd'hui, la tendance est d'accepter le fait que le partage des rôles est inévitable pour đEdipe à Colone. Cependant, la critique ne songe pas à considérer cette possibilité comme valable pour tout le répertoire.

Quant à nous, nous proposons ici d'accepter le partage de rôles de la même manière que nous acceptons la « règle des trois acteurs » : il s'agit d'une convention qui va à l'encontre de nos conceptions classiques du théâtre mais qui était en totale cohérence avec la conception grecque antique. Nous allons considérer la possibilité de partager un même rôle entre deux ou trois acteurs à chaque fois que cela nous permet d'expliquer des distributions compliquées, voire de rendre plus intéressante une distribution apparemment simple. Mais avant cela, examinons mieux les rôles que nous allons distribuer et éventuellement partager. 

réflexe qui consiste à assimiler le rôle au personnage qui est incarné un acteur tout au long de la représentation. Dans la tragédie antique, on peut établir une équivalence entre «rôle» et "personnage» (le rôle étant l'ensemble des apparitions d'un même personnage dans une pièce), mais il faut garder à l'esprit que les différentes parties qui composent ce rôle peuvent avoir des caractéristiques différentes d'une scène à une autre, et à titre, être jouées par différents acteurs. En d'autres termes: ce ne sont pas des «personnages » qui sont distribués entre les acteurs mais des « parties ». Pour constituer un rôle complet, le poète-metteur en scène disposait de trois acteurs et de figurants. Un rôle peut donc avoir des "parties» muettes mais, pour qu'il nous intéresse ici, il doit avoir au moins une partie parlée.$$
\text { de place, cest de considerer le type de vers qu'l comporte. La plupart des roles tragiques }
$$
de place, c'est de considérer le type de vers qu'il comporte. La plupart des rôles tragiques sont entièrement composés de vers iambiques, c'est-à-dire parlés. Très rarement, il arrive qu'un rôle soit entièrement composé de vers lyriques (chantés) ou de vers anapestiques (ce que conventionnellement on appelle "récités»). Plus souvent, un rôle comporte des vers iambiques et des vers anapestiques ou/et des vers lyriques dans des parties différentes d'une pièce. Nous pouvons donc considérer les rôles comme comportant ou non des parties parlées, chantées ou récitées ${ }^{17}$.

Une autre façon de considérer les rôles est de les classer selon leur importance dans la pièce. Il s'agit moins, bien entendu, de l'importance que le personnage a dans l'avancée de l'action, que de sa présence sur scène, de sa centralité dramaturgique, de son pathétique. La tragédie est un genre où l'action n'a pas forcément beaucoup de place, où un personnage qui ne fait que se lamenter peut avoir le rôle principal, et tel est souvent le cas. Pensons par exemple à l'Électre de la tragédie homonyme de Sophocle: une dramaturgie centrée sur l'action pourrait même éliminer ce rôle (ou, pour mieux dire, ne pas l'inventer), car Électre ne fait rien tout au long de la pièce, et choisir plutôt de se focaliser sur le fils qui accomplit l'action de tuer sa mère; dans la pièce de Sophocle, le rôle d'Oreste est pourtant largement subordonné à celui d'Électre. Un rôle principal n'a même pas besoin de subir un changement de sort après ses longues lamentations, que ce soit un changement positif comme celui d'Électre ou néfaste comme celui de Déjanire dans les Trachiniennes de Sophocle. Ainsi, la destinée malheureuse d'Hécube s'est-elle déjà réalisée au début des Troyennes d'Euripide, et elle ne fait guère qu'accumuler des motifs de pleurs. Le pathétique d'une partie doit donc être considéré comme un élément donnant du relief à l'acteur.

19 Une autre classification des rôles est celle qui s'attache aux « rôles-titre ». Très souvent le personnage central d'une tragédie coïncide avec son titre. Tel est le cas des Électre de Sophocle et d'Euripide, d'Edipe Roi et d'Ædipe à Colone de Sophocle, par exemple. Mais le titre peut également faire référence à l'un des personnages, non pas parce que c'est lui le principal de la pièce, mais parce que c'est la façon la plus naturelle de faire référence à sa trame. Par exemple, le rôle-titre du Rhésos pseudo-euripidéen a très peu de relief, mais c'est autour de la mort de ce personnage que s'articule l'argument de la pièce. Des considérations de ce genre doivent être faites pour des tragédies comme Agamemnon d'Eschyle ou Iphigénie à Aulis d'Euripide, voire pour l'Antigone de Sophocle.

Nous avons aussi les rôles typiques, ou des rôles comportant des parties typiques, abondants dans la tragédie grecque. La femme plaintive à laquelle nous faisions allusion en est un exemple. Elle pleure généralement un bonheur antérieur et un changement 
dans sa fortune; il peut s'agir d'une femme de haut rang qui tombe en servitude (Hécube, Cassandre, Andromaque), d'une épouse délaissée (Médée, Déjanire, Hermione, Créuse, Phèdre), d'une lamentation funèbre très emphatique (Électre, Évadné, la Muse), d'une exilée en terre étrangère et hostile (rôles-titre d'Hélène, Iphigénie en Tauride et Médée), voire d'une jeune femme qui doit mourir de façon prématurée (rôles-titre d'Antigone, Alceste, Iphigénie à Aulis, Polyxène dans Hécube, Électre dans Oreste, Cassandre). Ce sont des rôles d'un pathétique énorme et qui s'avèrent essentiels pour le genre tragique, à tel point qu'on les retrouve souvent aussi dans les chœurs comme " personnage collectif »: dans Les Troyennes d'Euripide et dans les deux Suppliantes.

Presque tous ces rôles comportent une partie lyrique. Ainsi, les personnages féminins ajoutent très souvent au pathétique le registre chanté. Au chant il faut joindre la danse, qui devait être parfois remarquable, comme dans les vers déchaînés de Cassandre dans Les Troyennes et dans Agamemnon ou d'Io dans Prométhée enchaîné. Les rôles masculins ayant ces caractéristiques sont beaucoup moins courants. Pour qu'un homme chante et pleure dans une tragédie comme le font souvent les femmes, il faut que sa chute soit particulièrement calamiteuse et toute récente, ou qu'il soit en proie à une douleur accablante ; c'est le cas du rôle-titre d'ÆEdipe Roi, de Créon dans Antigone, d'Héraclès dans Les Trachiniennes, du rôle-titre de Philoctète (toutes pièces de Sophocle), de Polymestor dans Hécube d'Euripide et de Xerxès dans Les Perses d'Eschyle.

Un autre rôle typique dans la tragédie est celui du messager. Il arrive que ce rôle corresponde à des personnages nommés, mais, dans la plupart des cas, ce sont des personnages anonymes, des serviteurs, des hérauts ou des citoyens de condition humble qui ont été témoins des faits qu'ils narrent. Les parties des messagers comprennent en général de longues tirades qui décrivent avec beaucoup de détails des faits affreux ou prodigieux, avec le sang-froid qu'implique une narration objective, mais sans détachement émotionnel pour autant. Ce sont des personnages mineurs qui en général n'ont aucun poids dans le déroulement de l'argument, mais à qui reviennent toutefois des parties qu'on considère comme essentielles, voire comme des morceaux de bravoure, dans une tragédie.

Les rois sont souvent présents dans les tragédies. Un lieu commun dans la littérature antique est que les rôles des rois étaient des parties sans grand relief quant au jeu d'acteur. Cela est sans doute vrai pour la plupart des rois de notre répertoire, qui ne sont pas impliqués dans la trame, ont des parties assez réduites, et sont souvent antipathiques, voire considérés comme le "méchant » de la pièce (le seul Ménélas est le roi méchant de cinq pièces). Certains rois, bien entendu, ne tombent pas dans cette catégorie, et sont des personnages centraux pleins d'importance dans la trame et dans la dramaturgie, comme Edipe dans $\Subset$ dipe-Roi, Créon dans Antigone, etc.

Les dieux sont aussi des rôles qui, en général, ne comportent pas de parties vraiment intéressantes pour un acteur. Ils sont présents dans plusieurs prologues d'Euripide pour expliquer la situation initiale et anticiper les faits (Hippolyte, Alceste, Les Troyennes, Ion), ou bien apparaissent à la fin de la pièce comme deus ex machina pour changer le déroulement des événements, expliquer des faits qui étaient restés jusqu'alors inconnus des personnages et/ou révéler des faits futurs (Oreste, Philoctète, Ion, Iphigénie en Tauride, Hippolyte, Hélène, etc.). La grande exception est le Prométhée enchainé, qui compte avec un personnel dramatique presque exclusivement divin. À part Prométhée, le seul dieu directement impliqué par les faits d'une tragédie humaine est la Muse de Rhésos, qui chante à la fin de la pièce la mort de son fils; mais sa participation dans la tragédie se 
réduit à cela et reste tout de même très brève. Une autre exception est Athéna dans Les Euménides, qui, même si elle n'est pas «touchée » par l'action, a le rôle le plus long de la pièce et chante avec le chœur dans un grand final unique dans le répertoire.

\section{Critères de distribution}

Cette variété de rôles doit donc être distribuée entre trois ou deux acteurs. Ces acteurs étaient organisés selon une hiérarchie qui impliquait que l'un d'entre eux devait avoir des parties capables d'en faire l'acteur principal. Cela devait être clair pour le public et surtout pour le jury qui, à partir de la deuxième moitié du ve siècle, choisissait à la fin des concours le meilleur acteur principal du festival. Le concours n'exigeait aucune différence nette entre les deux autres acteurs, mais il est possible qu'il y ait eu un écart quant à leur professionnalisme, leur prestige, leur talent et leur place dans les tétralogies. Ainsi, nous devrons établir des critères permettant d'évaluer les parties des tragédies d'une façon qui nous aide à décider s'il faut les imaginer comme des parties plutôt propres au premier, au deuxième ou au troisième acteur.

Plus un personnage a de relief dans une pièce, plus il a de pathétique, de centralité dramaturgique, de vers, de complexité poétique, musicale et chorégraphique, plus la probabilité est grande qu'il soit joué par le protagoniste. Mais rien n'oblige, $a$ priori, à attribuer un rôle entier à un seul acteur. Si un rôle est sans intérêt dramatique et poétique dans un épisode mais plein de pathétique et de vers chantés dans un autre épisode, nous devons considérer la possibilité de distribuer ces deux parties à deux acteurs différents: la plus intéressante au protagoniste, l'autre à l'un des acteurs secondaires. Il ne semble pas vraisemblable, par exemple, que l'acteur principal attende la fin d'une pièce pour faire montre de son talent, pendant qu'il laisse la prééminence aux deux autres acteurs tout au long de la tragédie.

La tendance que nous avons déjà critiquée parmi les spécialistes est d'essayer d'identifier le plus possible acteurs et personnages. Nous avons quant à nous plutôt des arguments pour les dissocier: plus un acteur principal joue de personnages différents dans une même pièce, plus il a l'occasion de montrer au public et au jury l'étendue de sa gamme de jeu et son habileté. La souplesse est encore aujourd'hui une qualité très appréciée chez les acteurs, et tout semble indiquer qu'elle l'était aussi, voire encore plus, dans l'Antiquité. Déjà les rhapsodes épiques en faisaient grand cas, en changeant leur style de récitation selon la partie récitée, et en « jouant » avec leur voix les différents personnages lorsqu'ils récitaient des vers en discours direct. Pour donner à l'acteur principal le plus grand nombre possible de parties "fortes", le partage des rôles entre plusieurs acteurs était fort utile. En outre, cet agencement des parties devait susciter un très grand intérêt dans le public; n'oublions pas que les trames des tragédies étaient connues des spectateurs et que les poètes prenaient souvent soin d'anticiper dans les prologues les innovations qu'ils pouvaient introduire dans les mythes, ce qui indique clairement que l'intérêt du public se portait plus sur la façon de présenter les faits, y compris le jeu des acteurs, que sur l'histoire elle-même.

Nous pouvons imaginer que certaines parties typiques étaient beaucoup plus convoitées par les protagonistes que d'autres ${ }^{18}$. Les femmes plaintives étaient sans doute considérées comme des parties fortes, tout comme celles des rois dont la chute représente le nœud de la trame. D'autres rois, en revanche, les typiques « porteurs de sceptre », ne présentaient 
aucun intérêt et étaient sans doute laissés aux acteurs secondaires. Les protagonistes ne devaient pas dédaigner les longs récits des messagers, et pouvaient donc incarner de simples serviteurs anonymes. Les dieux, par contre, devaient être considérés comme des rôles appropriés pour les acteurs de moindre importance. Quant aux personnages qui prêtent leur nom au titre de la tragédie, nous ne devons pas les donner au protagoniste sans évaluer auparavant les parties qu'ils comportent. Autrement dit, le prestige d'un rôle est évalué en termes poétiques et actoriaux; les classifications sociologiques des personnages n'entrent pas en ligne de compte dans l'attribution des parties.

La complexité métrique et la difficulté musicale doivent être considérées comme des éléments essentiels. Dans une tragédie, ce sont un ou deux personnages qui chantent, très exceptionnellement plus. Nous sommes tentés d'imaginer que les vers chantés sont toujours confiés au protagoniste. Or il arrive que, selon la distribution que nous pouvons reconstituer, nous ne puissions pas le faire. Cela est certain pour, au moins, une pièce de notre répertoire, comme nous le verrons plus bas. Que devons-nous en déduire? Que les parties chantées n'étaient pas toujours considérées comme les plus importantes? Cela semble en contradiction avec le fait que les parties lyriques devinrent avec le temps les favorites absolues du public et qu'on donnait des récitals de morceaux lyriques à la façon d'un récital d'airs d'opéra.

Pour comprendre les difficultés auxquelles nous faisons face quand nous voulons imaginer une reconstitution de la distribution d'une pièce, nous ne devons pas oublier que, pour tout le répertoire conservé, c'étaient les mêmes acteurs qui jouaient la totalité de la tétralogie d'un même poète, ou du moins le même acteur principal. Quand on évaluait le jeu d'un acteur, on évaluait sa performance dans l'ensemble de la tétralogie et non pas dans une pièce isolée. Or, à l'exception de l'Orestie d'Eschyle, nous ne conservons que des pièces isolées de leur contexte tétralogique. Si l'acteur devait faire montre de versatilité, de qualités vocales, de talents de danseur, etc., il devait le faire tout le long de la tétralogie et non pas forcément tout le long de la pièce. Cela pourrait expliquer que, dans une pièce donnée, le protagoniste ne semble parfois jouer qu'un seul rôle ${ }^{19}$, ou qu'il ne chante pas un seul vers lyrique ${ }^{20}:$ il avait en effet le temps de se rattraper dans l'une des autres pièces. On peut même imaginer que le poète pouvait composer des pièces où le protagoniste avait une place différente : un seul rôle principal dans une pièce, plusieurs rôles dans une autre, des parties plus parlées, des parties plus chantées...

\section{Les pièces}

Nous avons analysé la modalité de travail des acteurs de tragédie, puis les rôles qu'ils jouaient, puis proposé des critères de distribution de ces rôles parmi ces acteurs. Essayons maintenant d'appliquer ces critères aux pièces, en choisissant des tragédies qui puissent illustrer les différents types de distribution possible et qui, en même temps, permettent d'apprécier la différence entre notre façon de distribuer les rôles et celle adoptée par la plupart des critiques qui se sont occupés de la matière. Traditionnellement, on n'a guère pris en considération la prééminence d'un acteur sur les autres et on a ignoré la possibilité du partage des rôles. De même, les critères de distribution ne sont généralement pas conçus selon le jeu d'acteur demandé par les parties. seule pièce conservée où le nombre d'acteurs est semblable au nombre de personnages, est, pour cela même, la seule pièce pour laquelle, partant, on puisse avoir une distribution 
virtuellement certaine. Le roi de Thèbes, Étéocle, devait être joué par Eschyle lui-même, pendant que l'acteur assistant jouait le messager. Le rôle du premier acteur est nettement supérieur à celui du deuxième, mais le deuxième avait lui aussi une partie importante, notamment un récit de messager tout à fait remarquable.

Les choses sont plus compliquées pour n'importe quelle autre pièce. Prenons par exemple Les Perses d'Eschyle, tragédie qui est la plus ancienne conservée. Entre le poète et son seul acteur assistant, nous avons quatre rôles à distribuer. On a souvent proposé l'attribution des rôles de la reine Atossa et celui de son fils Xerxès au protagoniste ${ }^{21}$, l'argument étant que c'est à cause de cela que la reine quitte la scène avant l'arrivée de son fils - au motif d'une excuse assez faible sur le plan dramatique - pour ne plus y revenir. Le rôle de Xerxès, qui ne comporte pas un seul vers parlé (iambe), est d'un pathétique considérable ; c'est un grand rôle tragique qu'on doit attribuer au premier acteur, Eschyle lui-même. Mais le rôle de la reine est, en fait, le moins intéressant de la pièce. Le messager et le fantôme de Darius, qui partagent la scène avec elle, présentent sans doute plus d'intérêt. En conséquence, si nous attribuons Atossa au protagoniste, nous attribuons au deuxième acteur ces rôles, de sorte que nous n'avons pas d'acteur immédiatement identifiable comme l'acteur principal: les deux acteurs se partagent entre deux les parties les plus intéressantes de la pièce. $\mathrm{Si}$, au contraire, nous voulons un protagoniste net, nous donnerons le très long récit du messager - remarquable parmi tous les récits de son genre - et la scène spectrale du roi mort au premier acteur, tandis que le seul rôle de la reine reviendra à l'acteur assistant.

Que la tendance de la critique soit d'ignorer, quand on cherche à reconstruire les distributions, l'importance réelle que le rôle pouvait représenter pour le jeu d'acteur, se confirme si nous prenons le cas de l'Agamemnon d'Eschyle. Pour cette pièce - comme pour le reste de la tétralogie dont elle fait partie - Eschyle disposait déjà de trois acteurs. Le rôle-titre est généralement attribué au protagoniste. Mais quel intérêt présente ce rôle, très court, uniquement parlé, sans aucun pathétique, pour l'acteur principal ? En même temps, le rôle de Cassandre, plus long, plein de pathétique, de chant débridé, de danse... est généralement attribué au troisième acteur. Pourquoi ? Cassandre entre dans la scène avec Agamemnon, en présence de Clytémestre ${ }^{22}$; comme Agamemnon est censé avoir été attribué au protagoniste et Clytémestre au deutéragoniste, Cassandre doit être jouée par le tritagoniste. En établissant une telle distribution, disent souvent les critiques ${ }^{23}$, Eschyle a ajouté un effet formidable à la pièce: les spectateurs ne savaient peut-être pas qu'Eschyle allait utiliser un troisième acteur (innovation récente), et imaginaient jusqu'au dernier moment que Cassandre était incarnée par un figurant, vu qu'elle reste muette jusqu'à la sortie des deux autres acteurs et ce malgré les invitations à parler qu'on lui fait. Ainsi, quand elle éclate en des chants exaltés, la surprise du public devait être grande. L'effet serait en effet remarquable. Mais faut-il penser que le public ignorait combien d'acteurs allaient jouer dans la tétralogie de chaque poète? La question n'est pas mineure, car sans cet effet de surprise, l'attribution de Cassandre au troisième acteur semble sans fondement. Or il y avait, avant les concours, un événement appelé proagon (" avant-concours »), où le poète se présentait avec ses acteurs ${ }^{24}$.

Imaginons une distribution totalement différente. Tout d'abord, reléguons le rôle d'Agamemnon à un acteur secondaire, en raison de son manque d'intérêt pour l'acteur principal. Donnons à ce dernier plutôt le rôle de Clytémestre, qui est sans aucun doute le plus important de la pièce. Que faire de Cassandre, qui partage la scène avec la reine? Donnons-la aussi au protagoniste, en imaginant tout simplement que lorsque Clytémestre 
sort pour recevoir son époux, elle est jouée par un acteur secondaire. Après cette performance lyrique exceptionnelle, Cassandre entre dans le palais pour être assassinée, et le protagoniste reprend le rôle de Clytémestre. À ces deux rôles remarquables, on pourrait ajouter celui du Garde du prologue ; le résultat serait un premier acteur avec une prééminence absolue. L'acteur qui joue Agamemnon pourrait jouer aussi Égisthe en s'efforçant d'en bien différencier le caractère, ce qui aurait un effet intéressant et donnerait à cet autre acteur l'opportunité de montrer lui aussi sa souplesse. L'acteur restant jouerait le héraut et la partie de Clytémestre dans la scène avec Agamemnon et Cassandre.

La prééminence du premier acteur pourrait être conservée dans les autres pièces de la trilogie en lui attribuant les rôles d'Électre, de Clytémestre et de la Nourrice dans Les Choéphores et ceux de la Pythie, du fantôme de Clytémestre et d'Athéna dans Les Euménides . L'acteur qui a joué Égisthe et Agamemnon peut jouer Oreste et encore une fois Égisthe dans Les Choéphores, et encore Oreste dans Les Euménides et être imaginé ainsi comme un deuxième acteur avec un écart considérable par rapport au troisième. Ce dernier pourrait jouer Pylade et le Serviteur dans Les Choéphores et Apollon dans Les Euménides. Oreste est sans doute, lui aussi, un rôle digne du protagoniste dans Les Choéphores, mais non dans Les Euménides.

Mais la pièce où nous pouvons appliquer nos critères de distribution avec le plus d'intérêt est sans doute l'Antigone de Sophocle. L'avis de la critique a toujours été très partagé quant à la détermination du personnage principal de cette tragédie : le titre de la pièce et la popularité immense d'Antigone font qu'on se penche vers elle, tandis que tous les autres critères font préférer Créon. En effet, Créon apparaît aussitôt après la parodos, son édit - qui déclenche le conflit de la tragédie - ayant déjà été mentionné dans le prologue ; il clôt la pièce, il est présent presque dans toutes les scènes, il a de longs discours, des débats avec presque tous les personnages, des anapestes, des parties chantées, un pathétique hors pair, et c'est le renversement de son sort qui constitue le dénouement. Nous pourrions donc penser donner ce rôle au protagoniste. Mais voici que les sources qui ont été conservées nous orientent vers une piste contraire: Démosthène, dans un discours contre Eschine ${ }^{25}$, dit que son rival jouait toujours les troisièmes parties et, après avoir cité un fragment du premier discours de Créon en disant qu'Eschine l'a souvent récité, ajoute que les rois sont dans toutes les tragédies un privilège des tritagonistes ${ }^{26}$. Une partie de la critique a accepté ce renseignement sans le discuter et a attribué le rôle de Créon, malgré l'évidence dramatique, au tritagoniste. Une autre partie l'a ignoré absolument et a considéré Créon comme le rôle du protagoniste, tandis que quelques critiques, tout en préférant eux aussi cette distribution, ont essayé d'expliquer la contradiction avec l'affirmation de Démosthène. Nous allons présenter, d'après G. M. Sifakis ${ }^{27}$, une hypothèse qui propose une synthèse des deux alternatives.

La pièce s'ouvre avec Antigone, qui est le moteur de la trame de la tragédie. Pendant la plus grande partie de la pièce, c'est le personnage central. Dans sa dernière scène, elle chante des vers lyriques d'un grand pathétique qui confirment que son destin est loin d'être de faible importance dans le développement de la trame; elle ne disparaît pas en s'éclipsant sans laisser trace, comme c'est le cas d'Oreste dans Les Euménides. Tout cela fait d'Antigone un rôle qu'aucun protagoniste ne pourrait dédaigner. En fait, on peut facilement concevoir une Antigone qui arriverait jusqu'à la mort du rôle-titre et qui omettrait la catastrophe qui s'abat sur Créon; dans une telle tragédie, le rôle-titre serait sans aucun doute la partie du protagoniste, voire peut-être la seule partie du 
protagoniste. Mais tel n'a pas été le choix de Sophocle. Ce qu'il a créé est ce que la critique a appelé une "pièce en diptyque » : un personnage domine la scène jusqu'à un certain point, après quoi il disparaît (il meurt) et laisse la place à un autre personnage central. C'est aussi le cas des Trachiniennes avec Déjanire et Héraclès et d'Ajax avec Ajax et Teucer. La critique accepte généralement que les deux personnages centraux de ces deux autres pièces étaient joués par le même acteur. Nous pouvons même imaginer que l'intérêt des tragédies en diptyque était justement de donner à l'acteur principal deux rôles bien différents et faire de ces deux rôles des personnages de grand relief. Mais dans Les Trachiniennes comme dans Ajax, Héraclès et Teucer entrent en scène après la mort de Déjanire et d'Ajax respectivement, tandis que dans Antigone, Créon et le rôle-titre partagent la scène. Voilà pourquoi la critique n'a pas songé à attribuer les deux rôles au même acteur.

Considérons maintenant le rôle de Créon. Lui aussi peut être considéré comme un personnage "en diptyque ", tellement le renversement de son sort et de son âme est marqué. C'est un roi autoritaire, inflexible, opiniâtre, apparemment inébranlable jusqu'à son entretien avec Tirésias, après le départ d'Antigone ; dans toute cette première partie de la pièce, il ne parle qu'en iambes. Puis il sort de la scène, bouleversé par les paroles du devin, pour y revenir accablé de douleur, son image de gouvernant inébranlable absolument détruite; il parle alors avec des anapestes et des vers lyriques, ce qui implique une danse possiblement très prononcée. C'est un grand final d'un effet époustouflant, et on aurait du mal à croire que le protagoniste pouvait laisser une telle partie à un acteur secondaire. Autrement dit, nous sommes d'accord avec Sifakis pour dire que c'est lors de son retour que sa partie est nettement " protagoniste »; pendant le reste de la pièce, il serait difficile de soutenir que sa partie puisse disputer la prééminence à celle d'Antigone. D'ailleurs, les iambes cités par Démosthène (et c'est bien ainsi que l'orateur les appelle en les citant, « iambes », des vers parlés, non chantés) appartiennent au premier discours de Créon, dans la partie «non protagoniste » du personnage. Il n'y a alors pas d'incohérence entre les affirmations de Démosthène et l'évidence dramaturgique.

La proposition de Sifakis que nous reprenons ici est devenue, je l'espère, assez évidente : le protagoniste joue le rôle d'Antigone en entier, puis il revient dans le costume de Créon pour chanter et danser la scène finale. Pendant le reste de la pièce, Créon est joué par un acteur secondaire - le troisième acteur, si nous prenons le témoignage de Démosthène au pied de la lettre. À ces parties, le protagoniste peut même éventuellement ajouter celle d'Hémon avant le départ d'Antigone, et celle de Tirésias et d'Eurydice après son départ. C'est ainsi qu'il aura toutes les chances d'être un candidat imbattable pour le prix du meilleur acteur! Il joue la jeune héroïne rebelle qui s'oppose de façon farouche au statu quo, puis son jeune amoureux, passionné mais tout en retenue, puis un vieux devin aveugle, puis une mère bouleversée par une nouvelle concernant la vie de son fils, puis un roi livré aux pleurs et au désespoir qui arrive avec le cadavre de son fils dans les bras et apprend le suicide de sa femme, deux morts causées par lui-même. Quant au deuxième acteur, on peut lui attribuer les parties intégrales d'Ismène et du Garde, puis les récits bouleversants du Messager. Le tritagoniste ne jouerait que la partie iambique de Créon, ce qui le positionne désormais non seulement en dessous du protagoniste mais aussi du deutéragoniste. Une telle distribution concilie le témoignage de Démosthène avec les deux distributions que les critiques imaginaient - et tous avec de bonnes raisons - pour la pièce, tout en prenant en considération le fait qu'il n'y avait qu'un seul acteur qui 
participait au concours d'acteurs et qu'il devait donc avoir un éclat bien supérieur aux deux autres. Cette hypothèse se dégage de toutes les constatations qu'on peut faire et ne s'oppose à aucune. La seule raison pour laquelle elle n'a pas été accueillie par la critique est l'aversion envers le partage d'un rôle entre plusieurs acteurs; l'existence de ce partage étant assurée par d'autres pièces, cette aversion n'est pas justifiée, et il nous faut l'expliquer comme un préjugé basé sur des notions attachées au théâtre classique.

Nous pouvons considérer le cas d'Antigone comme paradigmatique d'un type de distribution où l'accumulation de plusieurs personnages et de passages lyriques donne au protagoniste sa place prééminente dans une tragédie. Le partage d'un rôle est non seulement ce qui rend possible cette accumulation, mais aussi ce qui met en évidence la sophistication des arrangements du poète en vue de l'éclat du premier acteur et du divertissement du public. Nous pourrions appliquer ces critères à d'autres pièces, comme nous l'avons fait avec Les Perses et Agamemnon. L'expérience donnerait une majorité de tragédies pour lesquelles cette espèce d'arrangement est possible, et dont une bonne part n'aurait de partie de protagoniste clairement prééminente qu'avec ces arrangements.

Il faut dire, cependant, que ces hypothèses sont loin d'être entièrement assurées. Nous avons plusieurs tragédies qui pointent vers d'autres directions. @Edipe-Roi, par exemple, où le rôle-titre est presque en tout analogue au Créon d'Antigone, mais avec cette différence que le rôle d'ÆEdipe doit être imaginé comme joué en entier par le protagoniste, sans qu'il ne joue aucun autre rôle. En effet, đdipe est le rôle central tout le long de la pièce et il est présent sur scène dans tous les épisodes. En outre, aucun autre personnage ne présente des traits plus intéressants que celui d'Édipe, pour qu'on essaie d'imaginer un agencement des parties beaucoup trop compliqué. Le cas du rôle-titre de Médée est semblable dans le sens où il devait être le seul rôle joué par le protagoniste, et ce, alors même qu'il s'agit d'un rôle sans vers lyriques : le protagoniste n'avait alors apparemment pas besoin de faire montre de souplesse ou de talents de chanteur pour mériter son titre.

Plus compliqué encore est le cas de l'Oreste d'Euripide. Grâce à des renseignements conservés dans certaines sources ${ }^{28}$, nous savons en effet que le premier acteur jouait le rôle-titre dès le début de la pièce. Aux parties de ce personnage, le protagoniste ne pourrait ajouter que la partie, assez réduite, du Messager. Le rôle d'Oreste est un grand rôle, sans doute, mais il ne chante pas un seul vers dans toute la tragédie. Et si, dans Médée , aucun personnage ne chantait, dans Oreste, en revanche, nous avons une quantité exceptionnelle de parties lyriques, pour les rôles d'Électre et du Phrygien. Qui plus est, ces deux rôles devaient être interprétés par le même acteur, que nous pouvons appeler deutéragoniste, et qui devait jouer aussi le rôle de Ménélas. Pour le tritagoniste resteraient les rôles d'Hélène, de Tyndare, d'Hermione, de Pylade et d'Apollon. Dans cette distribution, ce serait donc le deuxième acteur qui devrait se faire remarquer par son chant et le troisième qui ferait le plus montre de souplesse (même avec des rôles courts ou très courts). Il faut avouer qu'en suivant les principes que nous avons proposés auparavant, nous n'aurions pas supposé la distribution que les sources suggèrent. L'analyse de tout le répertoire nous propose donc ici un cas étonnant: des acteurs apparemment secondaires qui chantent pendant que l'acteur principal ne le fait pas, des parties très remarquables comportant des vers lyriques pour les deux acteurs secondaires, une pièce où aucune combinaison de parties ne semble donner un acteur principal intéressant... Pour les cas de ce genre, qui semblent mettre en échec l'existence d'un acteur prééminent, il faut cependant songer à ce qu'on a déjà rappelé : l'acteur 
principal l'était tout le long de la tétralogie du poète et concourait avec sa performance générale : une pièce unique ne peut suffire à juger son talent.

\title{
Conclusions
}

Une tragédie était donc une pièce de théâtre musical qui faisait partie d'une tétralogie ; cette tétralogie était toujours représentée lors d'un festival où elle participait à un concours. Pour l'énorme majorité des pièces conservées, ce concours consistait dans l'élection, par un jury, de la meilleure tétralogie d'une part, et du meilleur acteur principal d'autre part. Le spectacle général et le jeu du premier acteur étaient donc évalués de façon séparée. Mais l'indépendance entre la dramaturgie et la performance de l'acteur principal, tout comme dans un film d'Hollywood, ne peut être que relative. De même que le talent des trois acteurs d'une tétralogie devait contribuer d'une façon très considérable au succès d'une pièce, de même le poète devait avoir intérêt à donner à son acteur principal des parties qui lui permettent un éclat considérable. En outre, l'habileté avec laquelle il agençait ses trois acteurs et leur distribuait les rôles devait être une qualité appréciée par le public et par le jury. C'est pourquoi il semble important d'analyser les parties d'une tragédie en considérant à quel acteur chacune pouvait être destinée. Malheureusement, les sources nous indiquent l'importance de ces aspects sans nous permettre de comprendre avec clarté comment ils fonctionnaient effectivement. Malgré cela, ces considérations, combinées à la mise en jeu des conventions propres à la tragédie antique, peuvent changer de façon considérable la reconstitution d'un spectacle tragique à partir des textes conservés. En d'autres mots, la mise en scène d'une tragédie avec une distribution de trois acteurs nous rapprocherait beaucoup plus de la réalité spectaculaire du théâtre grec antique que les adaptations habituelles avec une distribution moderne.

\section{ANNEXES}

\section{Tragédies selon leur nombre de personnages}

\author{
2 personnages \\ Les sept contre Thèbes Esch. (Polynice, Messager) \\ 3 personnages \\ Suppliantes Esch. (Danaos, Roi, Héraut) \\ 4 personnages \\ Perses Esch. (Atossa, Xerxès, Messager, Fantôme de Darius)
}


5 personnages

Philoctète Soph. (Ulysse, Népotolème, Philoctète, Marchand, Héraclès) ;

Euménides Esch. (Pythie, Apollon, Oreste, Fantôme de Clytémestre, Athéna)

6 personnages

Électre Soph. (Pédagogue, Oreste, Électre, Chrysothémis, Clytémestre, Égisthe) ;

Agamemnon Esch. (Gardien, Clytémestre, Messager, Agamemnon, Cassandre, Égisthe) ;

Prométhée enchaîné Esch. ( ?) (Pouvoir, Héphaïstos, Prométhée, Océan, Io, Hermès)

7 personnages

Trachiniennes Soph. (Déjanire, Nourrice, Hyllos, Messager, Lichas, Vieillard, Héraclès) ; Choéphores Esch. (Oreste, Électre, Clytémestre, Nourrice, Serviteur, Égisthe, Pylade) ; Iphigénie parmi les Taures Eur. (Iphigénie, Oreste, Pylade, Thoas, Athéna, Pasteur, Serviteur) ;

Médée Eur. (Nourrice, Pédagogue, Médée, Créon, Jason, Égée, Messager) ;

Électre Eur. (Paysan, Électre, Oreste, Pédagogue, Clytémestre, Messager, Castor)

8 personnages

Antigone Soph. (Antigone, Ismène, Créon, Soldat, Hémon, Tirésias, Messager, Euridice) ;

Hécube Eur. (Polydoros, Hécube, Polyxène, Ulysse, Agamemnon, Polymestor, Talthybios,

Servante) ;

Hippolyte Eur. (Cypris, Hippolyte, Serviteur, Phèdre, Nourrice, Thésée, Messager,

Artémis) ;

Ajax Soph. (Athéna, Ulysse, Ajax, Tecmesse, Teucer, Ménélas, Agamemnon, Messager) ;

Edipe-Roi Soph. (Edipe, Prêtre, Créon, Tirésias, Jocaste, Messager corinthien, Esclave, Serviteur) ;

Edipe à Colone Soph. (ÆEdipe, Antigone, Ismène, Colonien, Thésée, Polynice, Créon, Messager) ;

Ion Eur. (Hermès, Ion, Pythie, Créuse, Xouthos, Serviteur, Pédagogue, Athéna) ;

Héraclides Eur. (Démophon, Alcmène, Iolaos, Macarie, Eurysthée, Serviteur, Héraut, Messager)

Troyennes Eur. (Athéna, Poséidon, Hécube, Cassandre, Talthybios, Andromaque, Ménélas, Hélène)

Suppliantes Eur. (Éthra, Thésée, Adraste, Évadné, Iphis, Messager, Héraut, Athéna); Héraclès Eur. (Amphitryon, Mégara, Lycos, Héraclès, Iris, Folie, Thésée, Messager) ; Bacchantes Eur. (Dionysos, Cadmos, Tirésias, Penthée, Serviteur, Messager, Agavé, Messager 2) ;

Iphigénie à Aulis Eur. (Iphigénie, Clytémestre, Achille, Agamemnon, Messager, Ménélas, Serviteur, Messager 2)

9 personnages

Alceste Eur. (Apollon, Trépas, Nourrice, Alceste, Admète, Eumélos, Phérès, Héraclès, Serviteur)

10 personnages

Oreste Eur. (Électre, Hélène, Hermione, Oreste, Ménélas, Tyndare, Pylade, Messager, Serviteur, Apollon) ;

Hélène Eur. (Hélène, Teucer, Ménélas, Gardienne, Théonoé, Theoclyménos, Messager Grec, Messager Égyptien, Serviteur, Castor) ;

Andromaque Eur. (Andromaque, Hermione, Ménélas, Fils d'Andromaque, Pélée, Oreste, Thétis, Servante, Nourrice, Messager) 


\section{1 personnages}

Rhésos Pseudo-Eur. (Hector, Paris, Énée, Rhésos, Dolon, Ulysse, Diomède, Athéna, Pasteur, Messager, Muse) ;

Phéniciennes Eur. (Jocaste, Serviteur, Antigone, Polynice, Étéocle, Créon, Ménécée, Tirésias, Messager, Messager 2, (Edipe)

\section{NOTES}

1. «Personnage collectif» dans le cas du chœur.

2. Les sources indiquent cela, mais elles aiment bien, en général, attribuer les grands changements à de grands noms.

3. V. Aristote, Poétique, 1449a. Anonyme, Vie de Sophocle, 23. Cf. Vie d'Eschyle, 13.

4. Il semble que la présence du poète sur la scène se soit maintenue jusqu'à ce que Sophocle l'abandonne à cause de la faiblesse de sa voix (Vie de Sophocle, 21).

5. Les chorèges prenaient en charge le payement des chorèges, tous les frais de préparation du spectacle, les costumes et tous les éléments matériels de la mise en scène. Les poètes, les acteurs et les joueurs d'aulos étaient, par contre, payés par la cité.

6. Les figurants n'étaient pas considérés comme des acteurs. Dans la plupart des pièces il faut considérer la présence d'un nombre assez large de personnages muets incarnant des suites, des esclaves, des soldats, des foules de citoyens, etc. Ils étaient costumés de la même façon que les acteurs.

7. La seule tragédie conservée où cela n'arrive pas est Les Sept contre Thèbes, mais même dans ce cas il faut penser que les tragédies se présentaient en tétralogies et la pluralité des personnages interprétés par chaque acteur était donc inévitable.

8. Voir, par exemple: Battezzato, Luigi, 2000, «The Thracian Camp and the Fourth Actor at Rhesus 565-691 ", Classical Quarterly 50, pp, 367-73. Même Prickard-Cambridge (1968, The Dramatic Festivals of Athens, deuxième édition revise par Gould et Lewis, Oxford) considère la possibilité de l'ajout d'un quatrième acteur.

9. Un parallèle clarifiant: un entraineur de football ne planifie pas une stratégie de jeu de manière abstraite, pour l'adapter ensuite aux joueurs dont il dispose.

10. Pendant tous les épisodes, Déjanire attend l'arrivée de son mari depuis longtemps absent. Mais quand Héraclès est près de revenir, Déjanire, par jalousie, provoque accidentellement la perte du héros ; quand ce dernier arrive agonisant dans l'exode, elle a déjà appris son malheur et s'est suicidée. Impossible sur le plan dramatique, la rencontre entre ces deux personnages l'est aussi sur le plan de la distribution, dès lors qu'un même acteur les prend successivement en charge. En outre, l'énorme écart entre le caractère du mari et la femme fait un effet de contraste remarquable, qui permet au protagoniste de faire valoir ces talents.

11. Des inscriptions qui servaient comme registre des concours indiquent le nom d'un seul des acteurs de chaque distribution, avec la simple étiquette d' « acteur ».

12. Plutarque, Praecepta Gererendae Reipublicae XXI 816f ; Cicéron, Discours contre Q. Cécilius 48 ; Démosthène, Sur l'ambassade, 245-250.

13. Quand la tragédie attique devient panhellénique et que des troupes d'acteurs jouaient des pièces de répertoire en tournée, l'acteur principal est devenule capocomico de la troupe. Voir, par exemple, Paulette Ghiron-Bistagne. Recherches sur les acteurs dans la Grèce antique, Paris, Belles Lettres, 1976.

14. Soulignons toutefois qu'il y a des personnages qui, bien que muets tout le long de la pièce, ont une identité bien définie et une présence très importante. C'est le cas notamment de Pylade dans 
les deux Électre de Sophocle et d'Euripide, et d'Iolé dans les Trachiniennes de Sophocle. Ils ne sont pas pour autant attribués à des acteurs.

15. La tradition critique suppose, depuis toujours, que le coryphée (chanteur principal du chœur) récite les parties iambiques tout seul - même Sifakis, sans y trouver de contradiction avec son concept de protagoniste comme interprète principal. Dans la mesure, toutefois, où les sources n'offrent aucun indice permettant de valider cette hypothèse, il nous semble qu'elle doit être mise en question, voire abandonnée.

16. Voir le tableau de synthèse présenté à la fin de cet article.

17. Il ne faut pas, cependant, surestimer le critère du vers. Tous les acteurs pouvaient chanter des vers lyriques, et dans certaines tragédies aucun n'en chante, les parties lyriques étant réservées au chœur.

18. Je préfère parler de "parties typiques» que de "rôles typiques». En effet, la partie de "femme plaintive " peut être appliquée à un rôle entier (Hécube des Troyennes) ou à un rôle qui, dans un autre épisode de la pièce, est constitué d'un autre type de partie (Hermione est la méchante de la première moitié d'Andromaque, puis devient une femme désespérée).

19. C'est le cas notamment des rôles titre d'Électre et d'‘Edipe-Roi de Sophocle.

20. Comme le rôle-titre de Médée.

21. Au moins depuis Kaffenberger, Heinrich (1911), Das Dreischauspielergesetz in der griechischen Tragödie, Darmstadt. Di Benedetto et Medda (1997, La tragedia greca sulla scena, Piccola Biblioteca Einaudi, Turin, p. 209) proposent même de considérer la Reine comme le seul personnage du protagoniste.

22. Forme transmise par les manuscrits, préférable au «Clytemnestre » traditionnel, faussement étymologique.

23. Marshall, C.W. (2003), « Casting the Oresteia », in The Classical Journal 98.3, pp. 257-274.

24. Pour le proagon, voir Pickard-Cambridge (op. cit.), p. 67 et ss.

25. Démosthène, Sur l'ambassade 245-250.

26. Une étude à paraître de Paul Demont analyse les implications de ce passage.

27. Op. cit., n2. Sifakis a été, en effet, un apport fondamental à mes hypothèses sur le partage des rôles et sur la prééminence du protagoniste. À ses hypothèses sur la distribution d'Antigone, j'ajoute certaines considérations.

28. Strattis, Anthroporestes, frag. 1 ; Aristophane, Les Grenouilles, 303.

\section{RÉSUMÉS}

La tragédie grecque avait des conventions très fixes qui la différencient nettement du théâtre européen moderne dont elle est souvent censée être la première manifestation. L'une de ces conventions concernait la distribution, qui était toujours composée de trois acteurs. Certaines des implications de cette convention sont évidentes pour un simple lecteur, mais d'autres, tout comme ses motivations, exigent une analyse et une réflexion plus approfondies. En nous basant sur les très maigres références antiques sur cette convention et en proposant des rapprochements avec d'autres manifestations culturelles, nous essaierons de démontrer que la juste appréciation de l'organisation des acteurs tragiques est indispensable pour une meilleure compréhension du genre. 
Greek tragedy, often claimed to be the first manifestation of modern European theatre, had very fixed conventions which distinguished it sharply from the latter. One of these conventions concerned the cast, which was always composed of three actors. Some of the implications of this convention are evident for a simple reader, but others call for a deeper analysis and reflection, as well as its motivations. Based on the scant ancient references, we will propose comparisons with other cultural manifestations, and try to show that a right assessment of the organisation of the actors of a tragedy is vital for a better comprehension of the genre.

INDEX

Keywords : actors, cast, contest, roles, tragedy

Mots-clés : acteurs, distribution, competition, rôles, tragédie

\section{AUTEUR}

\section{DIOMEDES GARIAZZO LECHINI}

Université Paris-Sorbonne 